\title{
Vascular changes responsible for the reduced effectiveness of obliteration of uterine arteries in women with advanced cervical cancer
}

\author{
Grzegorz Raba ${ }^{1}$, Kamil Szczupak ${ }^{2}$, Piotr Stabiszewski ${ }^{2}$, Wojciech Skibinski ${ }^{3}$ \\ ${ }^{1}$ Institute of Obstetrics and Medical Lifesaving, University of Rzeszow, Rzeszow, Poland \\ ${ }^{2}$ Hospital, Przemysl, Poland \\ ${ }^{3}$ Hospital, Sanok, Poland
}

Videosurgery Miniinv 2015; 10 (2): 229-232

DOI: 10.5114/wiitm.2015.52061

\begin{abstract}
Introduction: Haemorrhages from the genital tract remain a major threat to the life of patients with advanced cervical cancer. It is possible to achieve haemostasis by both surgical techniques and the procedure of endovascular uterine artery embolization. However, in some women with loco-regionally advanced cervical cancer the obliteration of the uterine arteries is not effective.

Aim: Evaluation of morphological changes in uterine arteries in patients with advanced cervical cancer and comparison of their changes with the achieved haemostatic effect of obliteration.

Material and methods: The prospective study included a group of 8 women with cervical cancer at a clinical stage of IIB to IIIC according to the FIGO classification. 3D quantitative coronary angiography (QCA) was performed before uterine embolization. The haemostatic effect of uterine artery embolization was compared with observed vascular changes.

Results: Mean uterine artery length in patients who achieved complete hemostasis: $39.5 \mathrm{~mm}$ - right uterine artery; $38.7 \mathrm{~mm}$ - left uterine artery. Mean uterine artery length in patients who achieved partial satisfactory haemostasis: $32 \mathrm{~mm}$ - right uterine artery; $30.5 \mathrm{~mm}$ - left uterine artery. Mean uterine artery length in patients who achieved unsatisfactory haemostasis: $10.5 \mathrm{~mm}$ - right uterine artery; $19 \mathrm{~mm}$ - left uterine artery.

Conclusions: Shortening of uterine arteries worsens prognosis of the haemostatic effect of their obliteration in patients with advanced cervical cancer.
\end{abstract}

Key words: cervical cancer, 3D angiography, uterine embolisation, quantitative coronary angiography (QCA).

\section{Introduction}

Haemorrhages from the genital tract remain a major threat to the life of patients with advanced cervical cancer $[1,2]$. In the case of failure of antihaemorrhagic treatment (local or systemic), the effect of haemostasis can be achieved by obliteration of uterine arteries. In a substantial number of patients, it is possible to achieve haemostasis by both surgical techniques and the procedure of endovascular embolization [3-5]. However, this method is not fully effective in all patients. In some women with loco-regionally advanced cervical cancer, the obliteration of the uterine arteries is not effective [6]. To date, no prognostic factors are known that might allow one to predict the outcome of surgery of obliteration of uterine arteries in terms of achieved

\section{Address for correspondence}

Grzegorz Raba MD, PhD, Institute of Obstetrics and Medical Lifesaving, University of Rzeszow, Rzeszow, Poland,

phone: +48 605038 395, e-mail: g.raba@plusnet.pl 
haemostasis in this group of patients. The problem results from the highly vascularized environment of the pelvis [7]. The blood supply within the cervical cancer tumour comes mainly from the uterine arteries and the ovarian arteries, through the fallopian tube branch of the ovarian artery [8]. Vascularization by the round and uterosacral ligaments, as well as rectal, obturator and pudendal arteries, which are the terminal ends of the internal iliac arteries [9], must also be accounted for. The presence of arterio-arterial anastomoses in the neoplastic tumour constitutes an essential element hampering the effect of haemostasis during vaginal bleeding. The main anastomoses with branches of the internal iliac arteries include [9]:

1. The aorta, through communications between:

a) the uterine artery and the ovarian artery,

b) the iliolumbar artery and arteriae lumbales inferioris,

c) the middle rectal artery and the superior rectal artery (a branch of the mesenteric artery),

d) the lateral sacral artery and the median sacral artery.

2. The external iliac artery, through communications between:

a) the obturator artery and the inferior epigastric artery ("corona mortis"),

b) the iliolumbar artery and the deep circumflex iliac artery.

3. The femoral artery, through communications between:

a) the inferior gluteal artery and the superior perforating artery (a branch of the deep femoral artery),

b) the inferior gluteal artery and the medial circumflex artery of the thigh,

c) the internal pudendal artery and external pudendal arteries.

An advanced neoplastic process in the pelvis minor as well as prior chemotherapy causes individual variations in individual patient vascularization. Therefore, in clinical practice in patients with advanced stage cervical cancer, qualification for the surgery of obliteration of uterine arteries is often made on the basis of discretionary criteria and experience of individual centres.

\section{Aim}

Evaluation of morphological changes in uterine arteries in patients with advanced cervical cancer and comparison of their changes with the achieved hemostatic effect of obliteration.

\section{Material and methods}

The prospective study included a group of 8 women with cervical cancer at a clinical stage of IIB to IIIC according to the FIGO classification, who completed radiochemotherapy treatment between 18 and 46 months before. The patients were qualified for uterine artery embolization because of bleeding from the genital tract. By the Selinger technique the sheath passage size $6 \mathrm{Fr}$ was applied. Pigtail type and JGFJ 4.0 gauges were applied to the abdominal aorta and next to the common iliac arteries. Intravenous non-ionic contrast agent with the density of $550 \mathrm{mg} / \mathrm{ml}$ was applied and then the flow in the internal iliac arteries and their branches was rated using 3D angiography. The measurement of the uterine arteries' length was done using QCA. The analysis covered radiographic changes in the radiological image of uterine arteries including their length. Then the embolization of uterine arteries was performed and their length was compared with the haemostatic effect achieved $24 \mathrm{~h}$ after embolization. The resulting haemostatic effect was divided into 3 groups: 1) complete haemostasis - complete cessation of bleeding $24 \mathrm{~h}$ postoperatively; 2) satisfactory haemostasis - reduction in sanitary towels use of at least $50 \%$ within $24 \mathrm{~h}$ postoperatively, compared to $24 \mathrm{~h}$ immediately preceding the surgery; 3 ) unsatisfactory haemostasis - sanitary towels use reduction of less than $50 \%$ within $24 \mathrm{~h}$ postoperatively, compared to $24 \mathrm{~h}$ immediately preceding the surgery.

In order to evaluate the number of used sanitary towels, patients made individual diaries $24 \mathrm{~h}$ before and $24 \mathrm{~h}$ after embolization of uterine arteries. Another method of blood loss estimation besides a subjective patient diary, such as haematocrit/haemoglobin concentration or weighing the pads, was not used because of their imperfection. For patients' quality of life a subjective patient diary was considered as the most adequate method. Informed patient consent was provided.

\section{Results}

In patients who achieved complete haemostasis, the uterine artery length was in the range 31-44 mm (mean: $39.5 \mathrm{~mm}$ - right uterine artery; $38.7 \mathrm{~mm}$ left uterine artery). In patients who achieved partial 
Table I. Comparison of the results of 3D angiography with the haemostatic effect of obliteration of uterine arteries in the study group

\begin{tabular}{|c|c|c|c|c|c|c|c|c|}
\hline Patient number & 1 & 2 & 3 & 4 & 5 & 6 & 7 & 8 \\
\hline Age [years] & 54 & 66 & 48 & 62 & 47 & 55 & 49 & 52 \\
\hline FIGO classification & IIIC & $\| \mathrm{lb}$ & $\| b$ & IIIC & $I I I b$ & $\| b$ & $\| I I b$ & IIIC \\
\hline Time after radiochemotherapy [months] & 23 & 18 & 31 & 46 & 37 & 24 & 39 & 42 \\
\hline Right uterine artery length [mm] & 33 & 42 & 41 & 18 & 26 & 42 & 38 & 3 \\
\hline Left uterine artery length [mm] & 31 & 38 & 44 & 21 & 33 & 42 & 28 & 17 \\
\hline Complete haemostasis & Yes & Yes & Yes & & & Yes & & \\
\hline Partial satisfactory haemostasis & & & & & Yes & & Yes & \\
\hline Unsatisfactory haemostasis & & & & Yes & & & & Yes \\
\hline
\end{tabular}

satisfactory haemostasis, the uterine artery length was in the range $26-38 \mathrm{~mm}$ (mean: $32 \mathrm{~mm}$ - right uterine artery; $30.5 \mathrm{~mm}$ - left uterine artery). In patients who achieved unsatisfactory haemostasis, the uterine artery length was in the range 3-21 mm (mean: $10.5 \mathrm{~mm}$ - right uterine artery; $19 \mathrm{~mm}$ - left uterine artery) (Table I).

\section{Discussion}

Proper qualification of women with advanced cervical cancer for obliteration of uterine arteries can reduce the failure rate of this method in the palliative treatment of bleeding. The key to a proper choice of the method of palliative treatment of bleeding in these patients may be assessment of individual changes in the length and course of uterine arteries before the obliteration procedure. In the study conducted, the effect of complete haemostasis was achieved by embolization of uterine arteries in patients with uterine artery length greater than $39 \mathrm{~mm}$. The method was ineffective in patients with uterine artery length lower than $19 \mathrm{~mm}$. It may be assumed that in the case of loco-regionally advanced cervical cancer, neoplastic infiltration alters the conditions of vascularization of the pelvis minor. The tumour infiltrating the parametrium may occupy the spaces running alongside the length of uterine arteries, which in turn causes their gradual shortening, thereby reducing the blood flow. Reduced blood flow in uterine arteries induces a compensatory flow increase in other vessels, as well as promoting neoangiogenesis. In such situations, the share of uterine arteries in the distribution of blood to the tumour area is reduced, which evidently reduces the effectiveness of the obliteration of uterine arteries in palliative management of bleeding. To date no method has been proposed to predict the effect of obliteration of uterine arteries in patients with advanced cervical cancer [10]. It is worth noting that this procedure also carries the risk of certain complications even in non-oncological patients, therefore requiring careful qualification of patients [11, 12]. This leads to a search for other palliative methods of controlling bleeding from the genital tract. In addition to palliative radiotherapy [13] or chemotherapy treatment [14], alternative methods are used such as topical application of zinc chloride paste [15], or compression by means of tamponade. In the present study, the use of the techniques of 3D angiography and QCA directly before uterine artery embolization allowed us to observe the relationship between the length of uterine arteries and the achieved effect of haemostasis. The more shortened the uterine arteries were, the lower was the observed effectiveness of embolization. The most important limitation of this study is its small study group consisting of 8 patients. Therefore, the results can only be considered preliminary, although they are of clinical interest. They indicate that the prognosis of effectiveness for uterine artery embolization based on measuring their length can reduce the failure rate of this method through better qualification of patients for embolization. The available literature lacks data to assess the dependence of the haemostatic effect of uterine artery obliteration on their length in patients with advanced cervical cancer. Therefore, further research into the effect of vascular changes in the pelvis minor on the effectiveness of obliteration of uterine 
arteries in patients with advanced cervical cancer is clinically justified.

\section{Conclusions}

Shortening of uterine arteries worsens prognosis of the haemostatic effect of their obliteration in patients with advanced cervical cancer.

\section{Conflict of interest}

The authors declare no conflict of interest.

\section{References}

1. Albu S, Grigoriu C, Vasiliu C, et al. The role of uterine artery embolization in cervical cancer - single case report. Maedica (Buchar) 2011; 6: 137-40

2. Sobiczewski P, Bidzinski M, Derlatka P, et al. Laparoscopic ligature of the hypogastric artery in the case of bleeding in ad vanced cervical cancer. Gynecol Oncol 2002; 84: 344-8.

3. Zeghal Souki D, Touhami O, Rajhi H, et al. Selective arterial embolization in case of bleeding in advanced cervical cancer. Tunis Med 2013; 91: 558-9.

4. Singh C, Gupta M, Tripathi R, et al. Successful use of transcatheter embolisation in an emergent life-threatening situation of bleeding from uterine arteriovenous malformation. BMJ Case Rep 2013; doi: 10.1136/bcr-2013-008730.

5. Pyra K, Wozniak S, Szkodziak P, et al. Uterine artery embolisation in massive vaginal bleeding in ectopic pregnancy: case report. Przegl Lek 2013; 69: 399-401.

6. Yu L, Tan GS, Xiang XH, et al. Comparison of uterine artery chemoembolization and internal iliac arterial infusion chemotherapy for the combining treatment for women with locally advanced cervical cancer. Ai Zheng 2009; 28: 402-7.

7. Cepni I, Ocal P, Erkan S, et al. Comparison of transvaginal sonography, saline infusion sonography and hysteroscopy in the evaluation of uterine cavity pathologies. Aust N Z J Obstet Gynaecol 2005; 45: 30-5.

8. Kwon YS, Roh HJ, Ahn JW, et al. Conservative adenomyomectomy with transient occlusion of uterine arteries for diffuse uterine adenomyosis. J Obstet Gynaecol Res 2014; DOI: 10.1111/ jog.12649.

9. Raba G. Unilateral recanalisation of hypogastric artery after ligation for postpartum haemorrhage treatment. Videosurgery Miniinv 2014; 9: 289-91.

10. Kim TH, Kim JY, Sohn DK, et al. A prospective observational study with dose volume parameters predicting rectosigmoid oscopic findings and late rectosigmoid bleeding in patients with uterine cervical cancer treated by definitive radiotherapy. Radiat Oncol 2013; 8: 28.

11. Lowenstein L, Solt I, Siegler E, et al. Focal cervical and vaginal necrosis following uterine artery embolisation. Eur J Obstet Gynecol Reprod Biol 2004; 116: 250-1.

12. Nakash A, Tuck S, Davies N, et al. Uterine sepsis with uterine artery embolisation in the management of obstetric bleeding. J Obstet Gynaecol 2011; 32: 26-9.
13. Kang HC, Shin KH, Park SY, et al. 3D CT-based high-dose rate brachytherapy for cervical cancer: clinical impact on late rectal bleeding and local control. Radiother Oncol 2010; 97: 507-13.

14. Adewuyi SA, Shittu OS, Rafindadi AH, et al. Cisplatin chemotherapy for haemostasis in bleeding cervical cancer: experience from a resource-poor setting. Niger Postgrad Med J 2010; 17: 122-7.

15. Yanazume S, Douzono H, Yanazume Y, et al. New hemostatic method using Mohs' paste for fatal genital bleeding in advanced cervical cancer. Gynecol Oncol Case Rep 2013; 4: 47-9.

Received: 16.02 .2015 , accepted: 15.03 .2015 